\title{
Electronic Signature Development and Verification System by using Biometric Selection
}

\author{
Mrinal Paliwal
}

\begin{abstract}
Electronic signatures as a method of identification and safety of electronic records are dictated by the Information Technology Act 2000. Electronic signature is an electronic token that connects the company to the data record. It is intended to validate and authenticate electronic documents. Validation relates to the accreditation method of the content of the paper, and authorization relates to the accreditation method for the transmitter.. Registration method is performed using publicsignal encryption; the signatory utilizes its private key to produce a digital paper record. The purpose of the paper is to guarantee that its actual information remains untouched. Its diverse nature has facilitated the production, storage, distribution and recovery of information by a simple, quicker, precise and comfortable method without contemporary text-based rules and regulations. This has resulted the universe to go internet, leading in turn to greater technological reliance, to boost the use of mobile technology in everyday lives. Progressively company operations in cyber space are held out, including interaction, formal information and commercial activities. The situation from document to digital job has been transformed. The supply for an operating digital structure for signatures for both private as well as government sectors has increased quickly in recent years. This research examined the world normal digital signature systems in order to ensure optimal safety for electronic systems and examined its likely implementations in different fields. The writers have studied them thoroughly. The research is focused on the prospective of information technology and the total data on digital sign.
\end{abstract}

Keywords - Non Repudiation, Encryption, Authentication, Hash Function, Key-Pair, Information Technology, Recognition.

\section{INTRODUCTION}

For any electronic transactions it is essential that information be authenticated, repudiated and verified. Egovernment e-business omits its standard papers into an edocument in modern globe of e-commerce. However, the interference of data and the stamp falsification have greatly increased. Thus, a digital crime can happen from company to the normal customer who copies a paper. The electronic signature system is used to ensure the authenticity and safety of digital information. Digital signatures can be delineated as electronic information authentication methods i.e. to check that the official paper obtained is from the requested sender and that its contents are not modified since it has been produced by the individual. Just as the markings, signatures or signatures act as authors of paper documents in the comprehensive system, so does the digital signature as authenticator of the electronic official paper. It generates the validity of every electronic record which the cryptographic signature user wishes to validate by attaching his digital signature to the electronic data document. The signature is a unique bit of information, certifying that the manuscript to which the signature is affixed was written by a claimant or otherwise approved. Sign encryption, encryption and authentication of messages are performed. The infrastructure made up of sets, policies and methods of role which is reliant on two sets of buttons: personal important and government key, is the best system adopted to ensure safety for digital signature. While this infrastructure is an acknowledged approach, it has certain disadvantages. This couple of buttons is generated with government important software (e.g. RSA). Malfeasance is intended as a signer for these customers ' personal important or license authority, and papers can readily be subscribed with this number. Shielding the unique keys of the described infrastructure is the main goal of "public key" infrastructure system. Private cards are commonly saved on a computer device with a unique code or PIN. A further method to safeguard personal and official documents is to place them on an intelligent board that must be bought from a reliable supplier and performed for signature. If the account holder drops his card for any purpose, he might wind up with unpredictable problems. Subconsciously anybody else receives the electronic card and wants to use a signer's personal button to enter a signal, it is difficult to see that the account holder isn't the real signatory and also that the letter has not been signed. A digital signature shows that the letter or record has not been changed in service after completion of the email or paper. Any recipient will not be in a state to modify, change, alter, or tamper with the document created by originator.

\section{RELATED WORK}

A brand new ID-based signature arrangement was introduced. Signers have to provide their respective fingerprint in the Key Generation Center during the time when signer is registering for key generation. The "public key" sequence is then transformed and the respective private key published by the KGC. It is compulsory for a signatory to attest his identity with his fingerprint to assert this personal button. During the scanning, the recipient reassembled the fingerprint using the government button. Afterwards, the signer is advised to draw his finger on the scene to suit the rebuilt image. While the account holder is identified by this scheme with his biometric, the signer is compelled to check. Another paper talks about a framework that produces a single key from its obtained body picture to the speaker and the recipient to register a signal silently. In 
combination with both the unique keys, the document's intended fingerprint is encrypted and decrypted. Signer or account holder gives the paper fingerprint itself and its Signing Key which is already encoded with a mixed buttons. A safe electronic certificate system was introduced where thumb vein is used as a hidden button rather than intelligent pad or customer verification password. This powerful system was asserted by Hitachi Ltd. for the Water Signature. Another paper talks about a biometric personal button with 512 copies of passport card stored within the system and the password has been used is protected by one way hash function. This published paper also discloses about a system implemented by combination of ras and dsa. Al-Khouri and J. Bal proposed to use three-factor digital identity authentication. They described a combination of "public key" with electronic card and biometric for accurate authentication. They termed this combination as trio technology. The benefit of this described combination of three technologies is that no one other than the owner or user can only use the electronic card. Recommended an information swap mechanism and internet email signature. In accordance with the Public and Private Key Pair guidelines, the secret key is stored online and coded with a security code known only to the account holder. A single code is produced to obtain this encryption key. A one-time password is produced for the entry of this private key employing by a hardware encryption unit and sent to the cellular phone of the signatory. The algorithm offers a great functionality and application requirements in cloud interaction, but does not sufficiently check to identify the exact identity of the signer. If the user has misplaced their cell phone, someone can access your personal key using your one time password popped on your mobile phone.

\section{PROPOSED SYSTEM}

Signature creation and inspection processes on the web platform are carried out in our suggested scheme. Every customer has a set of buttons, as we understand in "PKI", personal and "public key"s. On a main, safe computer, secret keys are collected. Our primary objective is to protect this private key and provide a highest feasible amount of safety so we can use a computer safety (HSM) unit on the software computer to accomplish this objective. In order to ingress this private key and register a signer paper, you need two-factor encryption - biometric identity and something you've got - a deliberately produced one-time key. This means that a customer can register if his biometric vein picture model is combined with the model earlier saved in the database and then return it to the portable signer to produce an one time key. Afterwards the signer transfers this to the software server for the validation procedure to finish. This key provides private interactions among both the signer and the recipient for a given meeting. Figure represents the situation for generating signatures. 1

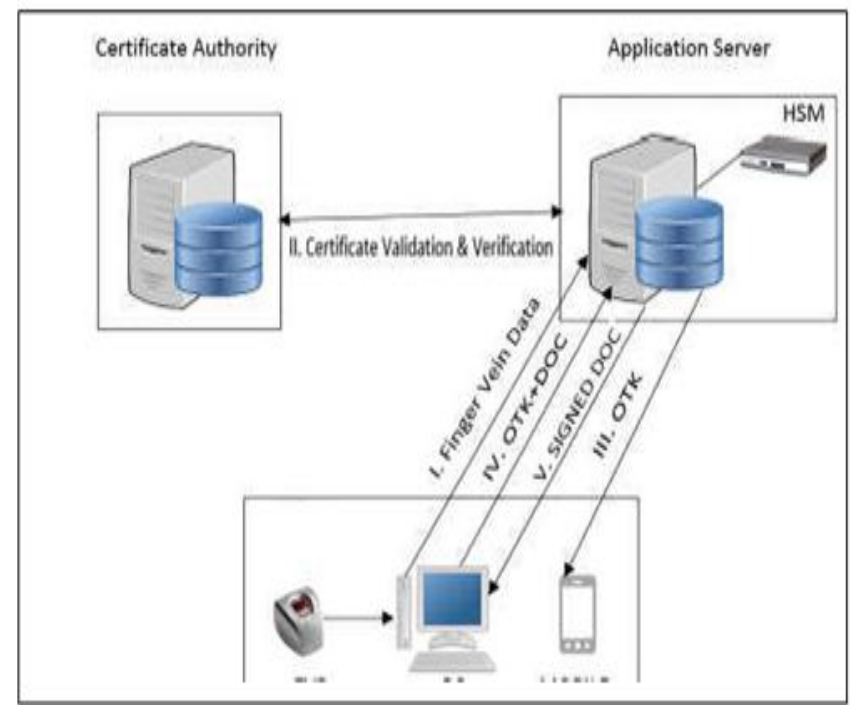

Figure 1 Signature Generator

Signer forwards his thumb print information to the software computer from the client terminal using a vein reader. With the assistance of the trusted root, the server handles and justifies the user certificates. When the registration method is completed, the server sends the registered paper to the user. The recipient must also provide his vein information to demonstrate his validity during pen control. This moment recipient only gives the client the one time key and the government authorized key to finish the checking method. The server then shows the recipient document's validation data. The notion of independently or from the server implementation of electronic signature was favorably regarded by the Forum of European Surveillance Authorities.

Fig. 1 explains about the key features and protocol employed in the system. The one time key used herein is a random integer; it uses mersenne twister protocol which produces crypto protected pseudo random integer. "Public key" used herein is md5 for fetching signed official paper context. Md5 is an encrypted protocol, coded by modified base64 protocol for super shielding the official papers, files and documents. The real signer does not recognize "PKI". When using biometrics instead of "PKI", specialized instruments are removed and the real signer is identified.

The real signer does not recognize "public key". When using biometrics instead of "public key" specialized instruments are removed and the real signer is identified. We indicate the model of vein as a bio metric and overcome all different biometric frauds. The biometric confirmation technique Finger Vein detects a person by using the vein model in a finger. Its fake acceptability level is one in a million, its fake refusal ratio is 1:10,000 and its default level is exceptionally small. Vein patterns are often blood-bearing bodies and so the only living human body can be authenticated. Deoxyhemoglobin is present in your blood and infrarouge lamps are consumed. The venous model is like black or dark markings and outlines. A unique camera picture of the vein motif is recorded by means of infrasound 
lamps. This picture is renewed into a model and contrasted during access control to the restored model.

\section{RESULT}

We can eradicate the possibility of fraud by using electronic signatures, even though the electronic signature cannot be modified. In addition it is difficult to forge a mark. We prove that this paper is legitimate with a electronic signature. We guarantee that the beneficiary is safe from incorrect data or forgery .The digital signing of the paper in issue satisfies some kind of legal necessity. Any legal element of the execution of the paper is covered by an electronic signature. Includes instant deadline and moment stamps that are crucial for corporate operations. This proposed system enhances the rate of transactions. Electronic signatures are an automated registration method that checks whether a parcel was sent by a particular person or company or that a paper was genuinely written by the user.

\section{CONCLUSION}

Regardless of the particular field execution of electronic certificates, encryption and information security are always the main aim. Furthermore, researchers interested in this field, sector have also drawn into consideration nonrepudiation, cost efficiency, effectiveness in moment, imposed sector norms, flexible, etc. The fresh horizon for implementation of digital signatures through objectorientated modeling is being studied each day, as the customer demands are increasing. This will also contribute to more strong electronic signature systems that are able to combat various kinds of crypto-system assaults. In addition, there are still several websites offering false identification including the "United States, Canada and Bangladesh." Our primary strategy is to create an enhanced digital signature system that meets present company requirements and meet user requirements, as well as to achieve a potential extension of the company's company requirements..

\section{REFERENCES}

1. Xiaodong Liu, Quan Miao and Daxing Li, "A New Special Biometric Identity Based Signature Scheme," International Journal of Security and its Applications, vol. 2, no. 1, Jan. 2008.

2. Ahmed B. Elmadani, "Trusted Document Signing based on use of biometric (Face) keys," International Journal of Cyber-Security and Digital Forensics, vol. 4, no. 1, pp. 289-296, 2012.

3. Successful development of biometric digital signature technology, available

at http://www.hitachi.com/New/cnews/130218.html Last accessed on 15-07-2016 at 7:09pm

4. Sambangi Eswara Rao and S.Ravi Kumar, "Novel Biometric Digital Signature System for Electronic Commerce Applications Using Java," International Journal \& Magazine of Engineering, Technology, Management and Research, vol. 1, no. 10, pp. 287-293, Oct. 2014.

5. A.M. Al-Khouri and J. Bal, "Digital Identities and Promise of the Technology Trio: "PKI", Smart Cards, and Biometrics," Journal of Computer Science, vol. 3, no. 5, pp. 361-367, 2007.
6. Wojciech Kinastowski, "Digital Signature as a Cloudbased Service," The Fourth International Conference on Cloud Computing, GRIDs, and Virtualization, 2013.

7. AyshaAlbarqi, EtharAlzaid, Fatimah Al Ghamdi, SomayaAsiri and JayaprakashKar, "“public key" Infrastructure: A Survey," Journal of Information Security, vol. 6, pp. 31-37, Jan. 2015.

8. Rachana C.R., "The Role of Digital Signatures in Digital Information Management," International Monthly Refereed Journal of Research in Management \& Technology, vol. 2, pp. 103-109, Mar. 2013.

9. Arulalan.V, Balamurugan.G and Premanand.V, "A Survey on Biometric Recognition Techniques," International Journal of Advanced Research in Computer and Communication Engineering, vol. 3, pp. 5708-5711, Feb. 2014.

10. Rupinder Saini and NarinderRana, "Comparison of Various Biometric Methods," International Journal of Advances in Science and Technology, vol. 2, pp. 24-30, Mar. 2014.

11. Dr. Rajinder Singh and Shakti Kumar, "Comparison of Various Biometric Methods," International Journal of Emerging Technologies in Computational and Applied Science, pp. 256-261, Feb. 2014.

12. Learn about "Barclays brings finger vein biometrics to internet banking", available at http://www.wired.co.uk/news/archive/2014-

09/05/barclaysfinger-scanner. Last accessed on 17-072016 at $11: 47 \mathrm{pm}$

13. Learn about "M2-FingerVeinTM - Non-invasive finger vein reader" available at http://www.m2sys.com/fingervein-reader/. Last accessed on 18-07-2016 at 03:00pm

14. Carl Ellison and Bruce Schneier (2000), "Ten Risks of "PKI": What You're not Being Told about "public key" Infrastructure," Computer security journal, vol. xiv, pp. $1-8,2000$.

15. Behrouz A. Forouzan, Data Communications and Networking, 4th ed., New York: McGraw-Hill, 2007.

16. Forum of European Supervisory Authorities for Electronic Signatures (FESA), "Public Statement on Server Based Signature Services," available at http://www.fesa.eu/publicdocuments/

PublicStatementServerBasedSignatureServices20051027.pdf.

17. Learn about Cloud Signing - Multiple Signing in Options available http://www.ascertia.com/Solutions/ByTechnology/cloudsigning

18. Secure CoSign Digital Signature Use via One-TimePassword (OTP) Authentication available at http://www.arx.com/files/documents/ cosigndigitalsignatures-and-otp.pdf 